\section{Aspects moléculaires de la mort subite de l'adulte}

Denis Escande

Inserm U.533, Institut

du thorax,

Faculté de Médecine,

CHU Hôtel Dieu,

1, place Alexis Ricordeau,

44093 Nantes Cedex 1, France.

denis.escande@

nantes.inserm.fr

> La cause la plus fréquente de décès dans les pays industrialisés demeure la mort subite liée à la maladie coronarienne. Dans la région de Maastricht [1], 1 décès sur 5 survenant à l'extérieur de l'hôpital est imputable à une mort subite. Dans $75 \%$ à $80 \%$ des cas de mort subite d'origine cardiovasculaire, le rythme cardiaque enregistré est la fibrillation ventriculaire. Dans $5 \%$ à $10 \%$ des cas, la mort subite survient en l'absence d'atteinte coronarienne ou d'insuffisance cardiaque. Plus rarement, l'origine de la mort subite est génétique, liée à la mutation de gènes impliqués dans l'électrogenèse cardiaque. Des maladies monogéniques de transmission mendélienne (Tableau I) sont à l'origine de morts subites.

\section{Torsades de pointes}

Ce trouble du rythme ventriculaire grave initialement décrit par F. Dessertenne [2] se distingue de la fibrillation ventriculaire par son caractère réversible et par son mécanisme. Toute altération allongeant la repolarisation cardiaque, mesurée par la durée de l'intervalle QT sur l'électrocardiogramme, peut entraîner des torsades-depointes, qui sont caractérisées par une variation rapide de l'axe électrique du cœur et par une baisse importante du débit cardiaque. La repolarisation des myocytes cardiaques étant sous la dépendance de courants potassiques, on comprend aisément que les mutations affectant les gènes codant pour les canaux potassiques du myocarde (KCNO1, KCNEl, KCNH2) et conduisant à une perte de fonction puissent être responsables d'un syndrome du PT long congénital. De la même façon, des mutations du canal sodique principal du myocarde, codé par le gène SCN5A, et conduisant à un gain de fonction, peuvent aussi être en cause. Notre laboratoire a récemment démontré la responsabilité, dans le syndrome du QT long congénital [3], du gène codant pour l'ankyrine $B$, une protéine d'ancrage de nombreux canaux ioniques $(\rightarrow)$.

On rapproche du syndrome $\quad(\rightarrow) \mathrm{m} / \mathrm{s}$ du QT long congénital la 2004, $\mathrm{n}^{\circ} 4$, prédisposition génétique p. 437 qui rend une proportion inconnue de la population générale particulièrement sensible aux nombreux médicaments bloquant de façon indésirable les canaux potassiques du myocarde et donnant lieu à des torsades-de-pointes ( $P T$ long acquis). Les anomalies génétiques à l'origine à cette hypersensibilité ne sont pas connues et pourraient être distinctes de celles affectant les gènes responsables du QT congénital.

\section{Tachycardie ventriculaire et fibrillation ventriculaire d'origine génétique}

Ces deux troubles du rythme mettent en jeu le pronostic vital et entraînent un effondrement du débit cardiaque. Contrairement à la torsade-de-pointes, la fibrillation ventriculaire (FV) est un trouble du rythme peu réversible. Elle peut être l'évolution ultime d'une tachycardie ventriculaire (TV) ou d'une torsade-depointe, ou survenir de façon inaugurale. Dans un contexte génétique, TV et FV peuvent être observées chez des patients dont le myocarde est structurellement normal ou, à l'inverse, structurellement anormal.
La survenue de TV et de FV en l'absence d'atteinte structurelle du myocarde peut être la conséquence d'un syndrome de Brugada [4], caractérisé par une surélévation du segment ST à l'ECG associée à un aspect de bloc de branche droite [5] (anomalie de la conduction électrique au sein du ventricule droit). Chez $15 \%$ à $20 \%$ des patients atteints du syndrome de Brugada, des mutations du gène SCN5A conduisent à une perte de fonction de la protéine, le gène morbide étant inconnu chez le reste des patients. Le mécanisme par lequel la perte de fonction du canal sodique engendre des troubles du rythme ventriculaire est encore discuté. On sait cependant depuis longtemps que les médicaments anti-arythmiques de classe I, qui bloquent les canaux sodiques, ont aussi, paradoxalement, un effet proarythmique marqué. Les TV catécholergiques surviennent typiquement à l'effort ou au cours du stress. Elles sont liées à des mutations du gène RyR2, qui code pour un canal calcique intracellulaire localisé dans la membrane du réticulum sarcoplasmique [6] (récepteur de la ryanodine), ou à des mutations du gène CASQ2, qui code pour la calséquestrine [7], une protéine de stockage du calcium dans le réticulum sarcoplasmique.

Les TV et FV survenant dans un myocarde structurellement anormal peuvent être liées à une dysplasie arythmogène du ventricule droit, pour laquelle plusieurs locus morbides ont été identifiés [8], ou à une cardiomyopathie hypertrophique ou dilatée [9], dont le potentiel arythmogène est bien connu et possiblement lié à une altération de l'énergétique cellulaire [10]. 


\section{Blocs de conduction}

\section{d'origine génétique}

Dès les années 1970, les enquêtes familiales réalisées chez des patients porteurs de blocs bifasciculaires ou de bloc complets ont suggéré la participation de facteurs génétiques dans la physiopathologie de formes rares de troubles conductifs [11]. En 1995, un premier locus morbide a été déterminé en 19q13.2-13.3 [12]. Plus récemment, notre laboratoire a identifié le premier gène impliqué dans des troubles conductifs progressifs dont le phénotype est proche de la description classique de la maladie de Lenègre [13, 14] (Encadré). Dans la famille étudiée, l'anomalie génétique consistait en une délétion de l'exon 22 du gène SCN5A, qui conduit à une pro- téine non fonctionnelle. La perte de $50 \%$ des protéines SCN5A fonctionnelles (haplo-insuffisance) conduit à des anomalies modérées à l'ECG, qui ne se révèlent qu'à l'âge adulte et ne donnent lieu que chez les sujets âgés à des troubles conductifs suffisamment sévères pour justifier l'implantation d'un pace-maker. Un même gène, plusieurs maladies Ainsi, le même gène SCN5A peut être responsable du syndrome du PT long (gain de fonction de SCN5A, risque associé de torsades-de-pointes), d'un syndrome de Brugada (perte de fonction de SCN5A, risque associé de fibrillation ventriculaire) ou d'un trouble isolé de la conduction (perte de fonction, risque associé de bloc auriculoventriculaire de haut degré, c'est-à-dire d'une interruption de la conduction nerveuse entre oreillettes et ventricules) (Encadré). Le plus étonnant est que ces syndromes sont capables de se combiner. Des mutations de SCN5A peuvent ainsi donner les associations suivantes: syndrome du QT long congénital + syndrome de Brugada [15], syndrome de Brugada + maladie de Lenègre [16], syndrome du QT long congénital + syndrome de Brugada + maladie de Lenègre [17].

\section{Qu'avons-nous appris ?}

L'électrophysiologie est une discipline particulière par le continuum d'informations qu'elle fournit à des niveaux très différents, moléculaire (patch-clamp), cellulaire (potentiels d'action) ou intégré ( $દ C G)$. Dans ce continuum d'informa-

\begin{tabular}{|c|c|c|c|c|c|c|}
\hline Syndrome & & Locus & Transmission & Gène & Protéine & Fonction \\
\hline \multicolumn{7}{|l|}{ QT long congénital (LQT) } \\
\hline \multirow[t]{7}{*}{ Syndrome de Romano-Ward } & LQTl & $11 p 15.5$ & $A D$ & KCN@1 & KvLQTl & Courant IKs \\
\hline & LQT2 & $7 q 35-36$ & $A D$ & KCNH2 & HERG & Courant IKr \\
\hline & LQT3 & $3 p 21-23$ & $A D$ & SCN5A & $\operatorname{SCN} 5 \mathrm{~A}$ & Courant INa \\
\hline & LQT4 & $4 q 25-27$ & $A D$ & $A n k B$ & Ankyrine B & Ancrage \\
\hline & LQT5 & $21 q 22$ & $A D$ & KCNEl & $\operatorname{minK}$ (ou IsK) & Courant IKs \\
\hline & LQT6 & $21 q 22$ & $A D$ & KCNE2 & MiRP & $?$ \\
\hline & LQT7* & $17 q 23-24$ & $A D$ & KCNJ2 & Kir2.1 & Courant IKI \\
\hline \multirow[t]{2}{*}{ Syndrome de Jervell et Lange-Nielsen } & JLN1 & $11 p 15.5$ & $A R$ & KCN@1 & KvLQTl & Courant IKs \\
\hline & JLN2 & $21 q 22$ & $A R$ & KCNEl & mink (ou IsK) & Courant IKs \\
\hline \multirow[t]{2}{*}{ Syndrome de Brugada (SB) } & SBl & $3 p 21-23$ & $A D$ & SCN5A & SCN5A & Courant INa \\
\hline & SB2 & $3 p 22-25$ & $A D$ & $?$ & $?$ & $?$ \\
\hline \multirow[t]{2}{*}{ Blocs de conduction héréditaires $(\mathrm{BCH})$} & $\mathrm{BCHl}$ & $19 q 13.2-13.3$ & $A D$ & & & \\
\hline & $\mathrm{BCH} 2$ & $3 p 21-23$ & $A D$ & SCN5A & SCN5A & Courant INa \\
\hline \multicolumn{7}{|l|}{ Tachycardie ventriculaires } \\
\hline \multirow[t]{2}{*}{ catécholergiques (TVc) } & TVcl & lq42.1-q43 & $A D$ & RyR2 & Ryanodine R & Calcium \\
\hline & TVc2 & lpl3.3-pll & $A R$ & CASP2 & Calséquestrine & Calcium \\
\hline \multirow[t]{2}{*}{ Fibrillations auriculaires familiales (FAF) } & FAFl & $10 q 22-24$ & $A D$ & $?$ & $?$ & $?$ \\
\hline & FAF2 & $11 p 15.5$ & $A D$ & KCNOI & KvLQTI & Courant IKs \\
\hline \multicolumn{7}{|l|}{ Dysplasie ventriculaire droite } \\
\hline \multirow[t]{7}{*}{ arythmogène (DVDA) } & DVDAl & $14 q 23-24$ & $A D$ & $?$ & $?$ & \\
\hline & DVDA2 & $1 q 42.1-q 43$ & $A D$ & RyR2 & Ryanodine R & Calcium \\
\hline & DVDA3 & $14 q 12-q 22$ & $A D$ & $?$ & $?$ & $?$ \\
\hline & DVDA4 & $2 q 32.1-q 32.3$ & $A D$ & $?$ & $?$ & $?$ \\
\hline & DVDA5 & $3 p 23$ & $A D$ & $?$ & $?$ & $?$ \\
\hline & DVDA6 & 10p12-pl4 & $A D$ & $?$ & $?$ & $?$ \\
\hline & $\begin{array}{l}\text { Maladie } \\
\text { de Naxos }\end{array}$ & $17 q 21$ & $A R$ & PLAK & Plakoglobine & Adhérence \\
\hline
\end{tabular}

Tableau I. Troubles du rythme et de la conduction d'origine génétique. AD: transmission autosomique dominante; AR : transmission autosomique récessive; calcium : protéine impliquée dans le métabolisme intracellulaire du calcium. HERG : human ether a-go-go related-gene; IKl: inward rectifier $\mathrm{K}^{+}$current; IKr, IKs: rapidly or slowly activating delayed rectifier $\mathrm{K}^{+}$current; INa: inward $\mathrm{Na}^{+}$current; Kir: inward rectifier potassium channel, 2.1 (famille 2, sous famille 1); KvLQTl: voltage-gated potassium channel LQTl; MinK: minimal potassium channel; MiRP: minK-related protein. *LPT7 est associé à une paralysie périodique et à un dysmorphisme dans le cadre du syndrome d'Andersen. 


\section{LES BLOCS AURICULOVENTRICULAIRES}

Dans ces troubles, l'influx électrique partant des oreillettes ne peut arriver aux ventricules ou y arrive avec retard, il est bloqué :

- dans le bloc auriculoventriculaire de $1^{\text {er }}$ degré, l'influx est simplement ralenti dans son trajet de l'oreillette vers le ventricule. Ce phénomène se traduit graphiquement sur l'électrocardiogramme par un allongement de l'espace PR (délai entre la dépolarisation des oreillettes et celle des ventricules);

- dans le bloc auriculoventriculaire de $2^{e}$ degré, l'influx est ralenti de plus en plus au fil des contractions, jusqu'à ce qu'intervienne une disjonction électrique, et ainsi de suite: ce sont les périodes de Luciani-Wenckebach ;

- dans le bloc auriculoventriculaire de $3^{e}$ degré, l'influx n'arrive jamais aux ventricules. Les oreillettes battent pour leur compte, les ventricules battent lentement à leur rythme : c'est la maladie du pouls lent permanent.

La maladie de Lenègre est une affection dégénérative survenant chez des patients âgés de plus de 60 ans. L'évolution vers un bloc auriculoventriculaire paroxystique ou permanent est possible, avec des épisodes de syncopes imposant l'implantation d'un stimulateur cardiaque.

tions, les maladies génétiques affectant le rythme cardiaque permettent de faire le lien entre altérations moléculaires et troubles cliniques. La perte de fonction du gène $K C N \rho 1$, qui code pour un canal potassique repolarisant, conduit très logiquement à un syndrome du QT long

congénital. De la même façon, une mutation avec gain de fonction de KCNOI conduit très logiquement, du fait du raccourcissement des périodes réfractaires atriales (de l'oreillette), à des fibrillations auriculaires familiales [18]. L'analyse des causes génétiques de la mort subite a confirmé la responsabilité des anomalies du métabolisme intracellulaire du calcium dans la genèse des troubles du rythme graves ventriculaires (gènes RyR2, CASQ2, AnkB...). Elle a aussi confirmé ce que nous savions, c'est-àdire que les troubles du rythme pouvaient naître de troubles de la conduction (SCN5A).

Les troubles du rythme (tachycardie ventriculaire) d'origine génétique responsables de morts subites ne touchent qu'une petite fraction des patients atteints de troubles du rythme qui, dans leur grande majorité, sont liés à des pathologies acquises. Néanmoins, cette analyse génétique éclaire singulièrement la physiopathologie des troubles du rythme et permet d'élaborer de nouvelles stratégies thérapeutiques. En pratique clinique, elle permet d'identifier et de traiter les patients à risque dans une fratrie, et en cela de sauver des vies. $\diamond$ Molecular aspects of sudden cardiac death

\section{RÉFÉRENCES}

1. De Vreede-Swagemakers JJ, Gorgels AP, et al. Out-ofhospital cardiac arrest in the 1990's: a populationbased study in the Maastricht area on incidence, characteristics and survival. J Am Coll Cardiol 1997 ; $30: 1500-5$.

2. Dessertenne F. La tachycardie ventriculaire à deux foyers opposés variables. Arch Mal Cœur 1966 ; 9:263-5.

3. Mohler PJ, Schott JJ, Gramolini A0, et al. Ankyrin-B mutation causes type 4 long-QT cardiac arrhythmia and sudden cardiac death. Nature $2003 ; 421$ : 634-9.

4. Brugada P, Brugada J. Right bundle branch block, persistent ST segment elevation and sudden cardiac death: a distinct clinical and electrocardiographic syndrome. A multicenter report. J Am Coll Cardiol $1992 ; 20: 1391-6$.

5. Wilde AA, Antzelevitch C, Borggrefe M, et al. Proposed diagnostic criteria for the Brugada syndrome: consensus report. Circulation 2002 ; 106 : 2514-9.

6. Priori SG, Napolitano C, Tiso N, et al. Mutations in the cardiac ryanodine receptor gene (hRyR2) underlie catecholaminergic polymorphic ventricular tachycardia. Circulation $2001 ; 103$ : 196-200.

7. Lahat $H$, Pras $\varepsilon$, Olender T, et al. A missense mutation in a highly conserved region of CASQ2 is associated with autosomal recessive catecholamine-induced polymorphic ventricular tachycardia in Bedouin families from Israel. Am J Hum Genet 2001 ; $69: 1378-4$.

8. Danieli GA, Rampazzo A. Genetics of arrhythmogenic right ventricular cardiomyopathy. Curr Opin Cardiol 2002 ; 17 : 218-21.

9. Roberts R, Schwartz K. Myocardial diseases. Circulation 2000; 102 (suppl 4) : IV3 4-9.

10. Javadpour MM, Tardiff JC, Pinz I, et al. Decreased energetics in murine hearts bearing the R92 $\rho$ mutation in cardiac troponin T. J Clin Invest 2003; $11: 768-75$.

11. Morgan CM, Gray KE, Robb GH. A survey of familial heart block. Br Heart J 1974 ; $36: 693-6$.

12. Brink PA, Ferreira A, Moolman JC, et al. Gene for progressive familial heart block type I maps to chromosome 19q13. Circulation $1995 ; 91$ : 1633-40.

13. Schott JJ, Alshinawi C, Kyndt F, et al. Cardiac conduction defects associate with mutations in SCN5A. Nat Genet $1999 ; 23: 20-1$.

14. Probst V, Kyndt F, Potet F, et al. Haploinsufficiency in combination with aging causes SCN5A-linked hereditary Lenegre disease. J Am Coll Cardiol 2003 ; $41: 643-52$.
15. Bezzina C, Veldkamp MW, van Den Berg MP, et al. A single $\mathrm{Na}(+)$ channel mutation causing both long-PT and Brugada syndromes. Circ Res 1999 ; $85: 1206-13$.

16. Kyndt F, Probst V, Potet F, et al. Novel SCN5A mutation leading either to isolated cardiac conduction defect or Brugada syndrome in a large French family. Circulation 2001 ; 104 : 3081-6.

17. Grant AO, Carboni MP, Neplioueva V, et al. Long QT syndrome, Brugada syndrome, and conduction system disease are linked to a single sodium channel mutation. J Clin Invest 2002 ; 110 : 1201-9.

18. Chen YH, Xu SJ, Bendahhou S, et al. KCNQl gain-offunction mutation in familial atrial fibrillation. Science 2003 ; 299 : 251-4. 